\title{
Design of a double dipole electron spectrometer
}

\author{
Antoine Maitrallain ${ }^{\mathrm{a}}$, Bas van der Geer ${ }^{\mathrm{b}}$, Marieke de Loos $^{\mathrm{b}}$, Grace Manahan ${ }^{\mathrm{a}}$, S. Mark \\ Wiggins ${ }^{\mathrm{a}}$, Wentao Li $^{\mathrm{a}}$, Mohammed Shahzad ${ }^{\mathrm{a}}$, Roman Spesyvtsev ${ }^{\mathrm{a}}$, Giorgio Battaglia ${ }^{\mathrm{a}}$, \\ Gregory Vieux ${ }^{\mathrm{a}}$, Enrico Brunetti ${ }^{\mathrm{a}}$, and Dino A. Jaroszynski ${ }^{\mathrm{a}}$ \\ ${ }^{a}$ SUPA, Department of Physics, University of Strathclyde, Glasgow, G4 ONG United Kingdom \\ and The Cockcroft Institute, Sci-Tech Daresbury, Keckwick Lane, Daresbury, Warrington WA4 \\ $4 \mathrm{AD}$, United Kingdom \\ ${ }^{\text {b} P u l s a r ~ P h y s i c s, ~ E i n d h o v e n, ~ T h e ~ N e t h e r l a n d s ~}$
}

\begin{abstract}
With the increase of laser power at facilities reaching petawatt-level, there is a need for accurate electron beam diagnostics of the laser wakefield accelerator (LWFA), which are becoming important tools for a wide range of applications including high field physics. Electrons in the range of several $10^{\prime} \mathrm{s}$ of $\mathrm{GeV}$ are expected at these power levels. Precise diagnostic systems are required to enable applications such as advanced radiation sources. Accurate measurement of the energy spread of electron beams will help pave the way towards LWFA based free-electron lasers and plasma based coherent radiation sources. We propose an innovative double dipole spectrometer suitable for characterizing bunches produced using a petawatt class laser.
\end{abstract}

Keywords: Electron spectrometer, diagnostic, simulation

\section{INTRODUCTION}

The laser wakefield accelerator (LWFA) ${ }^{1}$ is a very promising way of producing high quality electron beams ${ }^{2-4}$ at $\mathrm{GeV}$ level energies. ${ }^{5}$ This has lead to the need for very high precision, broadband, single-shot electron spectrometers $^{6-8}$ for characterizing the beam properties. Measuring the electron bunch spectral characteristics is of particular importance for developing free-electron lasers (FELs) ${ }^{9-15}$ for instance. For this type of application, a sub-percent energy precision is required. ${ }^{12,16}$

Most electron spectrometers use a magnetic element (a dipole) to deflect the charged particles and disperse them according to their energies through the Lorentz force $\vec{F}_{L}: \vec{F}_{L}=q_{e} \overrightarrow{v_{e}} \wedge \vec{B}$ where $q_{e}$ is the charge and $\overrightarrow{v_{e}}$ the velocity of the electron and $\vec{B}$ the magnetic field through which the particle travels; and a single detector screen, which is usually a scintillating screen $\left(\operatorname{Lanex}^{6}\right.$ or Ce:YAG ${ }^{7}$ ) or an array of fibers. ${ }^{8}$ In this case, the initial angle of the electron beam in the dipole is unknown, which can lead to an error in determining the energy. This effect can be mitigated using a collimator ${ }^{17}$ which allows the reduction of the incoming angle uncertainty but has the disadvantage of decreasing the charge and increasing the emittance. An imaging system (magnetic ${ }^{18,19}$ or a plasma lens ${ }^{20,21}$ ) can reduce this effect for a given energy range at the cost of a lower charge and a larger footprint. In our design, it is proposed to use two strictly similar dipoles that have reversed polarizations, and two sequentially placed detection screens, to simultaneously measure, in a single shot, both the incoming angle of the electron beam and its spectrum.

We have used the GPT (General Particle Tracer, Pulsar Physics http://www.pulsar.nl/gpt ${ }^{22}$ ) software tool to study this configuration. A number of simulations have been carried out to investigate this scheme within the experimental constraints (mainly the chamber size and electron targeted energy) and establish a viable design suitable for diagnosing $\mathrm{GeV}$ electron beams with a percent level energy error. RADIA ${ }^{23}$ simulations have been conducted to design the magnetic structure itself. These simulations and design will be presented. The final spectrometer will be installed and used in the new SCAPA (Scottish Centre for the Application of Plasma-based Accelerators) facility. ${ }^{24}$

Further author information: (Send correspondence to A. Maitrallain, Dino A. Jaroszynski)

A. Maitrallain: E-mail: antoine.maitrallain@strath.ac.uk

Dino A. Jaroszynski: E-mail: dino@phys.strath.ac.uk 


\section{PRINCIPLE AND DESIGN STUDY}

\subsection{Principle}

The double dipole electron spectrometer is sketched in Fig. 1. An electron beam is incident at an angle $\alpha_{0}$ on the first dipole of length $L_{1}$ and magnetic field $B_{1}$. To the first order, the Lorentz force bends the beam through an angle $\alpha_{1}+\alpha_{0}$, determined at the exit of the first dipole and entrance of the second one. This can be described by the equation $L_{1}=R_{1} \sin \left(\alpha_{1}+\alpha_{0}\right)-R_{1} \sin \left(\alpha_{0}\right)$, where $R_{1}$ is the Larmor radius of the electron due to the first dipole. The same equation can be used for the second dipole : $L_{2}=R_{2} \sin \left(\alpha_{1}+\alpha_{0}\right)-R_{2} \sin \left(\alpha_{2}\right)$, where $R_{2}$ is the Larmor radius of the electron due to the second dipole. Here the gyroradius of the electron $R_{i}$ is defined as $R_{i}=\frac{\gamma_{e} m_{e} v_{\perp, e}}{q_{e} B_{i}}$, where $\gamma_{e}, m_{e}$ and $v_{\perp, e}$ are the electron Lorentz factor, mass and component of the velocity perpendicular to the magnetic field respectively. In the case where both dipoles are strictly similar (geometrically and in terms of magnetic fields) but have reversed polarities, then $L_{1}=L_{2}$ and $B_{1}=B_{2}$ (hence $R_{1}=R_{2}$ ), the exit angle $\alpha_{2}$ of the electron beam is identical to the incoming angle : $\alpha_{2}=\alpha_{0}$.

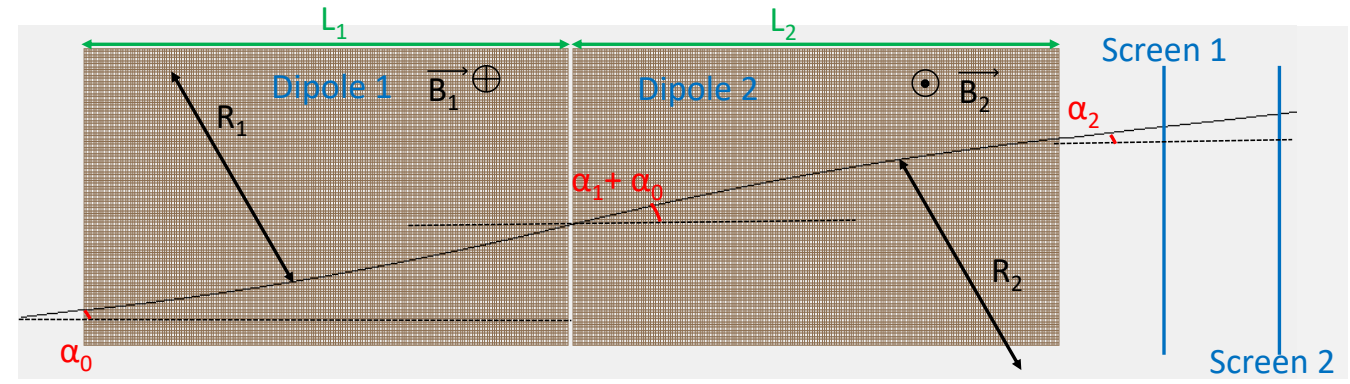

Figure 1. Sketch of the principles of the double dipole spectrometer.

The angle of the beam can therefore easily be obtained using two screens, a set-up commonly implemented in previous experiments. ${ }^{25-27}$ Assuming the dispersion is in the horizontal direction, the vertical angle of the electron beam is straightforward to determine only looking at the first screen. Spatial features can be inserted into the electron beam path, as in, ${ }^{25}$ an iterative approach ${ }^{26}$ or a closed set of equations ${ }^{27}$ can be used to retrieve the horizontal angle on a second screen in case of a broad spectrum. The measure of both the angle and spectrum allows resolving the energy with a very high precision. Knowing both the energy and angle is also of interest for high precision emittance measurements. ${ }^{28,29}$

Given the geometry of the experimental chamber, the following constraints are taken into account in the simulations: the source-dipole entrance distance $D_{S D}$ is $100 \mathrm{~mm}$ and the source-screen distance $D_{S S}$ is 1350 $\mathrm{mm}$. Subsequently the parameters chosen for the simulations are a magnetic field close to $1 \mathrm{~T}$ and an electron beam source of energy ranging from 50 to $1450 \mathrm{MeV}$ with a divergence up to $5 \mathrm{mrad}$. The gap in between the poles is chosen to be $20 \mathrm{~mm}$ in order not to block the radiation emitted from the laser plasma interaction region (betatron radiation, for instance). All the simulations were ran on a 3D particle tracking code, GPT, which includes space charge models.

\subsection{Comparison between the double dipole and single dipole, single screen schemes}

For equivalent dipoles the dispersion curves and errors associated with respect to the initial divergence are given in Fig. 2. For these results the dipoles used are strictly identical: a length of $400 \mathrm{~mm}$, a magnetic field of $1 \mathrm{~T}$ and in the two dipoles scenario, the distance between them is zero. It can be seen, first, that the low energy part of the curves are very similar, indeed these electrons are bent such that they do not enter the second dipole so their trajectory are identical in both cases. Whereas, there is clearly a different trend on the high energy part of Fig. 2a) and b), which shows electrons that pass through both dipoles.

The error is calculated by fitting the dispersion curve obtained in each case with a non divergent beam. Then by considering a given divergence the difference in the trajectories is calculated and compared with the first case, which gives us the energy error. Considering Fig. 2c) and d), it can be seen that the error on the energy is greater (by less then a factor 3 at $1 \mathrm{GeV}$ ) in the double dipole scenario for all divergences considered. The small 

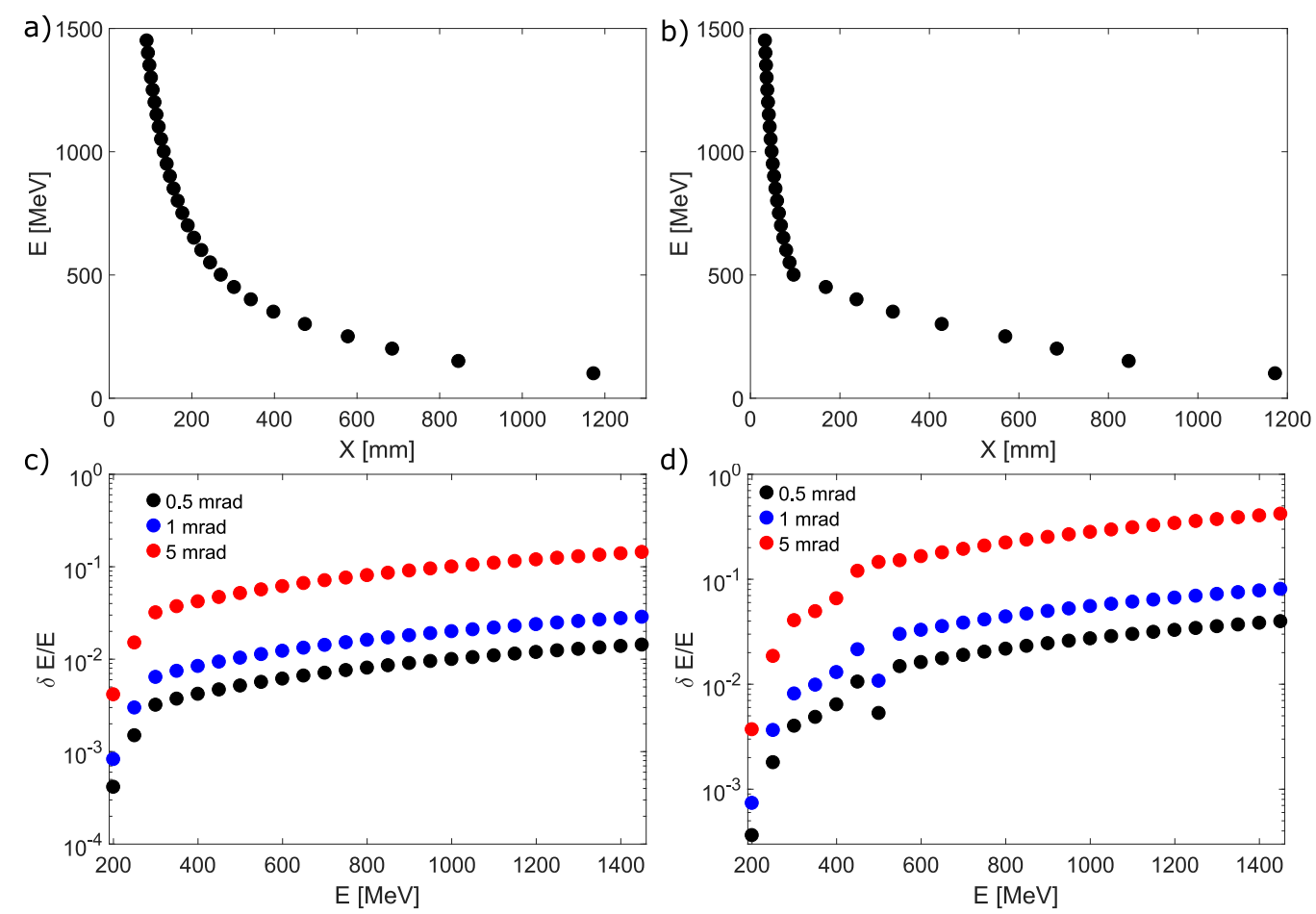

Figure 2. Comparison between the single and double dipole schemes : dispersion curves a) and b), and error associated with the incoming angle in logarithmic scale c) and d). For these results the parameters used were $D_{S D}=100 \mathrm{~mm}$ and $D_{S S}=1350 \mathrm{~mm}$, the dipoles used in both cases are strictly similar and the distance between both dipoles in the second case is set to $0 \mathrm{~mm}$.

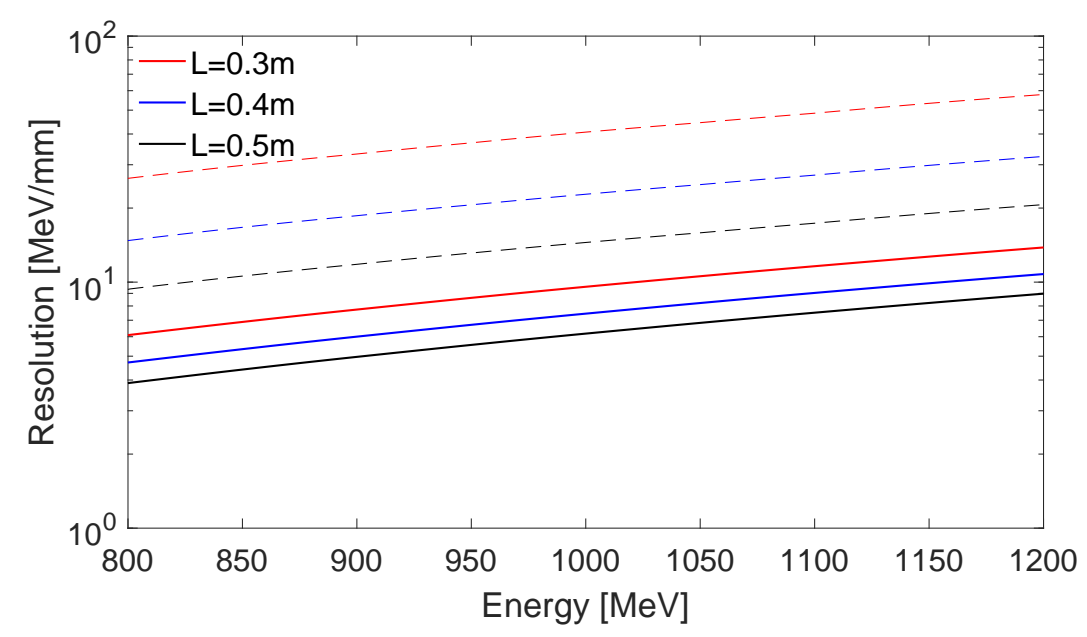

Figure 3. Resolution of the spectrometer as a function of the electron energy for different spectrometer length in the single dipole (thick) and double dipole scheme (dashed lines) case in logarithmic scale.

discontinuity of the curves in Fig. 2d) is due to the difference in the trajectory between electrons with different energies, for very low energy electrons that only pass through one dipole, compared with higher energy electrons that travel through the full length of both dipoles.

The resolution for different dipole lengths in both cases can also be compared, as shown in Fig. 3. The spectrometer resolution is clearly always better in the single dipole case (thick lines). This is because the 
geometrical dispersion of the double dipole is always less than in the single dipole case (dashed lines).

\subsection{Double dipole design study}

So far all simulations for the double dipole case have used two dipoles directly one after the other. Fig. 4 shows the effect of varying the distance between the two dipoles on the resolution at $1 \mathrm{GeV}$ (a)) and the error on the energy measurement for two distances $(10 \mathrm{~cm}$ in b) and $30 \mathrm{~cm}$ in c)). By increasing the distance between the dipoles, not only the resolution increases (by about a factor 2 in the distance range considered here) but the error on the energy measurement given the initial divergence also decreases by about $50 \%$ in this case.

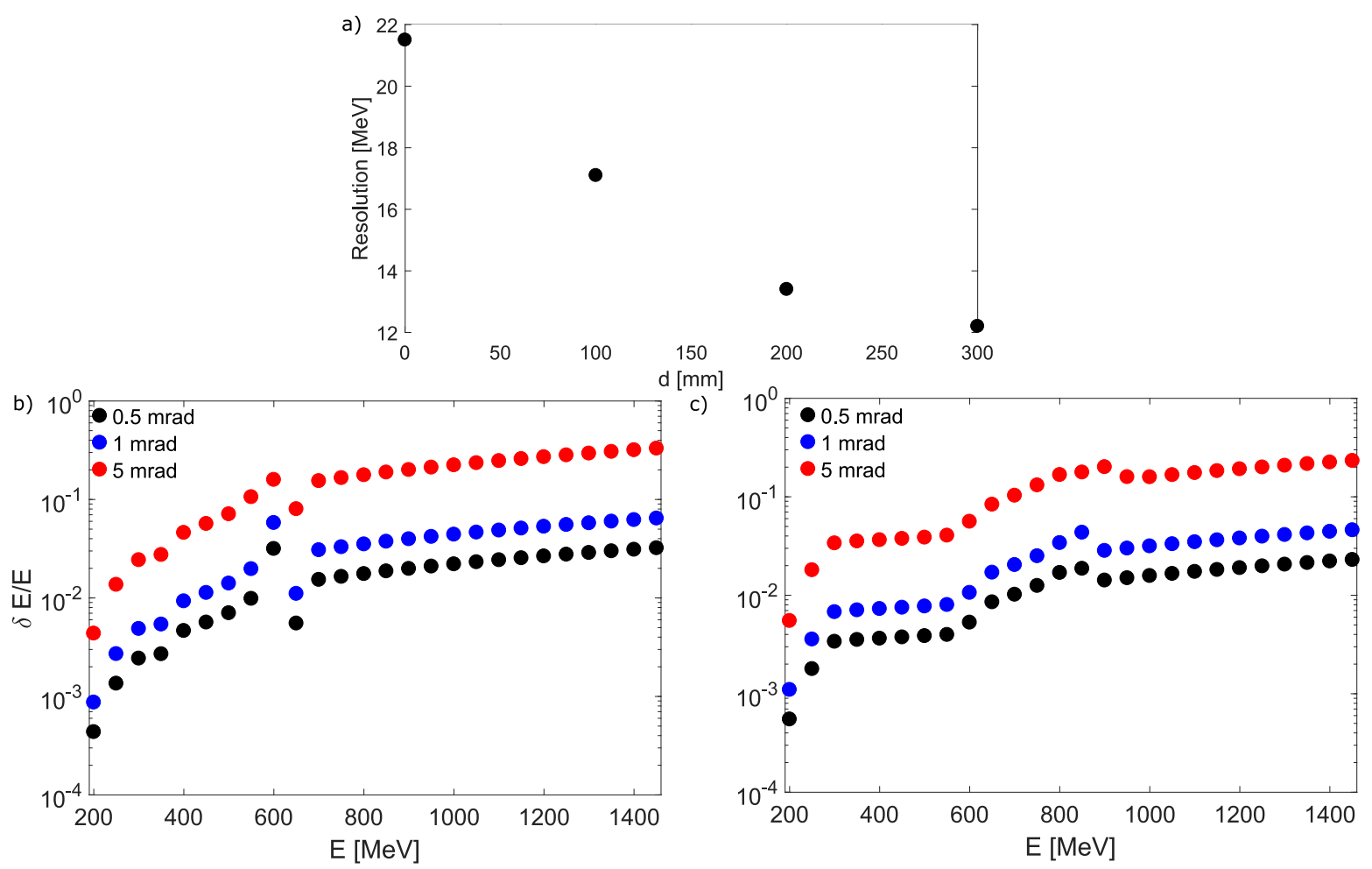

Figure 4. Effect of the distance on the double dipole spectrometer design. Resolution at $1 \mathrm{GeV}$ as a function of the distance between the dipoles a). Error on the energy measurement as a function of the electron energy for a distance of $10 \mathrm{~cm} \mathrm{~b}$ ) and $30 \mathrm{~cm} \mathrm{c}$ ) in logarithmic scale.

Although increasing the distance between the dipoles is promising, this cannot be increased arbitrarily because of constraints imposed by the experimental chamber size. In the following, a distance of $30 \mathrm{~cm}$ has been chosen to maximize the performances of the spectrometer while keeping the footprint reasonable. In this case, given the results on Fig. 4c), a double dipole, double screen scheme is necessary to achieve a very high precision (less than $1 \%$ at $1 \mathrm{GeV}$ ) energy determination for a beam divergence of about $0.5 \mathrm{mrad}$ or above.

\subsection{Double dipole and double screen spectrometer}

A schematic of the experimental set-up using the double dipole and double screen scheme is shown in Fig. 5. Examining the electrons trajectory in this figure enables a better understanding of the dispersion curve in Fig. 2b): low energy electrons (lower than $500 \mathrm{MeV}$ in this configuration and given the dipole width) do not pass through the second dipole, which explains the discontinuity in this curve.

When using several screens it is important to consider the interaction between the electron beam and the first screen. This has been studied previously and 3 main effects need to be taken into account: 


\section{Screen 3}

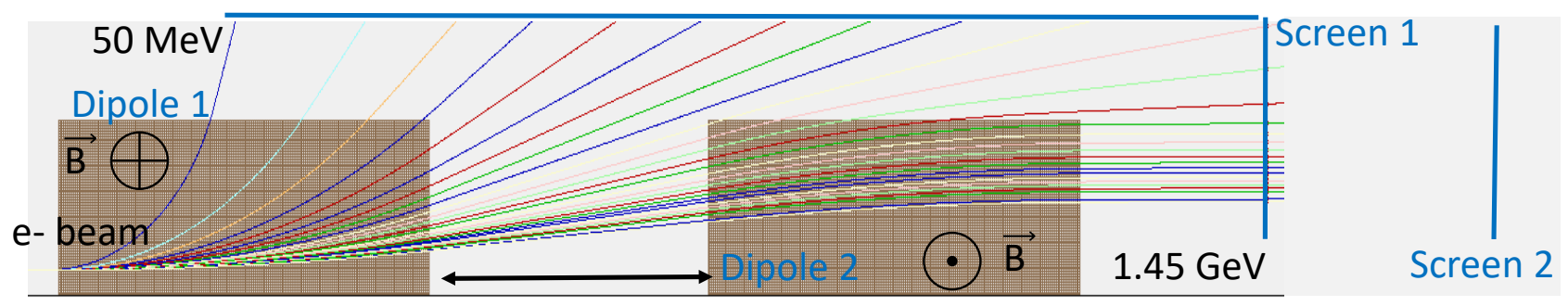

Figure 5. Schematic and electrons trajectory for the double dipole double screen set-up.

- the divergence increase due to Coulombian diffusion ${ }^{26,30}$

- the electron energy loss in the screen has been studied in ${ }^{31}$ using to Monte Carlo (MC) simulations

- the increase in energy spread as the electrons pass through the screen.
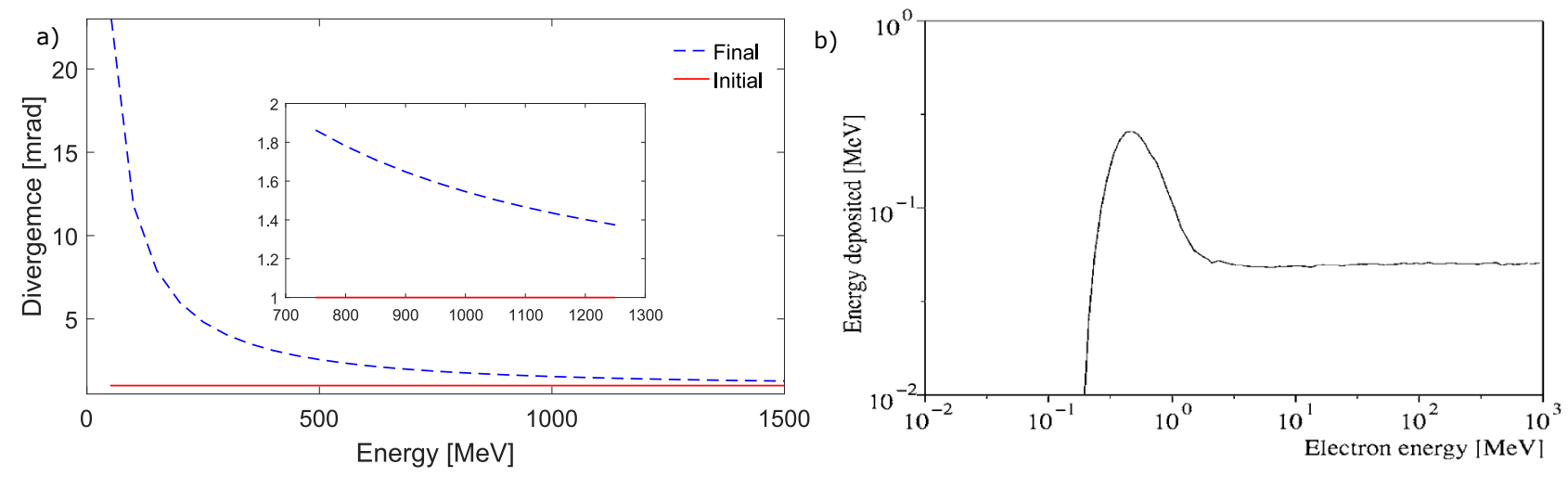

Figure 6. Final divergence a) and energy b) ${ }^{31}$ due to scattering in a $300 \mu \mathrm{m}$ thick Lanex screen on the electron beam with respect to its energy. The inset in a) is a zoom of the region of interest in our case. For a), the initial divergence of the electron beam is $1 \mathrm{mrad}$.

The increase of the divergence and the effect of energy loss are shown in Fig. 6. Fig. 6a) exhibits that the divergence grows significantly at $1 \mathrm{GeV}$ (see inset), for an initial divergence of $1 \mathrm{mrad}$, but since this can be calculated, it can be taken into account before estimating the beam horizontal incoming angle on the second screen. It can also be seen in Fig. 6b) that the mean energy loss is extremely small compared with the initial energy, therefore even if there are fluctuations around this average, the induced energy dispersion is negligible. This has been verified with Fluka ${ }^{32} \mathrm{MC}$ simulations. It can be explained by the fact that radiative effects are dominant in this regime but the thickness of the Lanex is very small (a few hundreds $\mu \mathrm{m}$ at most) when compared with the radiation length, consequently this effect can be neglected.

\subsection{Robustness of the system}

If the double dipole screen is to be used it needs to be a robust system. The sensitivity to small discrepancies that may occur while fabricating the two dipoles should not dramatically modify the performances of the spectrometer. Fig. 7 shows the output/input angle difference as a function of the energy for magnets of different lengths (as a fraction of the total length) in Fig. 7a) and for a comparable field difference in Fig. 7b). Firstly, there is a clear difference in trend between the electrons that travel through the full length of both dipoles $(\approx 750 \mathrm{MeV}$ 

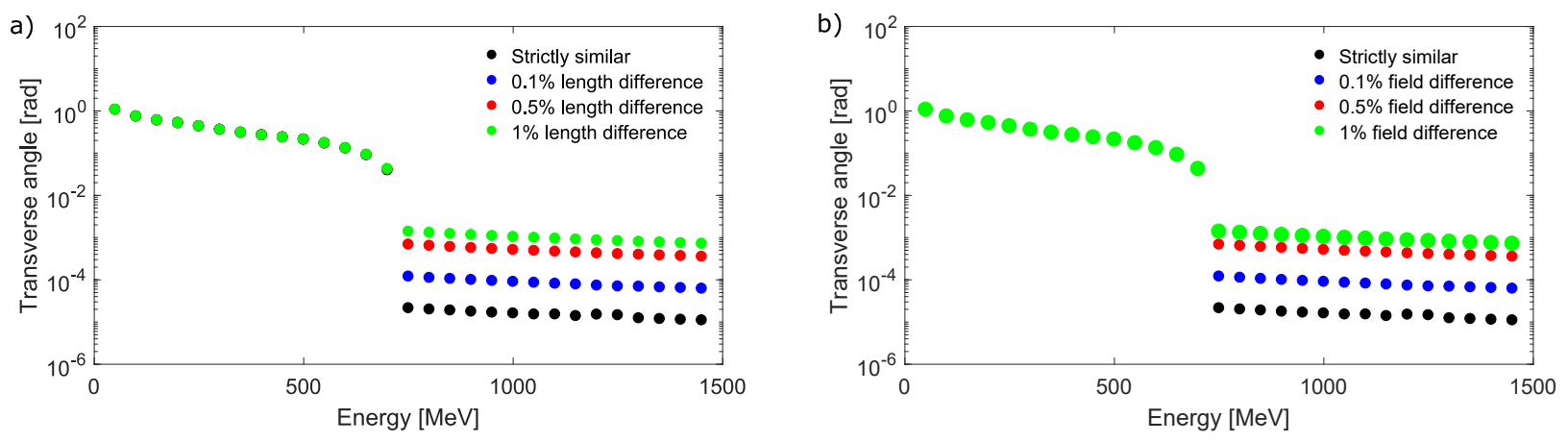

Figure 7. Difference between incoming and output angle as a function of the energy given a length a) and field b) difference between the two dipoles in logarithmic scale.

and above) and the other electron for which the deviation induced by the first dipole is not compensated for by the second one, resulting in a non zero output angle.

This figure shows a maximum induced output angle of about $1 \mathrm{mrad}$ at $1 \mathrm{GeV}$. This would be in the case of a $1 \%$ difference in length or field, in other word a $0.4 \mathrm{~mm}$ or a $0.1 \mathrm{~T}$ difference between both dipoles, which is very large given the manufacturing standards. The same type of study has been conducted adding noise to the original field map and the same type of conclusion is drawn. This study implies that an accurate field map will be required in order to precisely quantify the induced angle in case of discrepancies between the dipoles.

\section{OTHER USES OF THE SPECTROMETER}

\subsection{Energy selection}

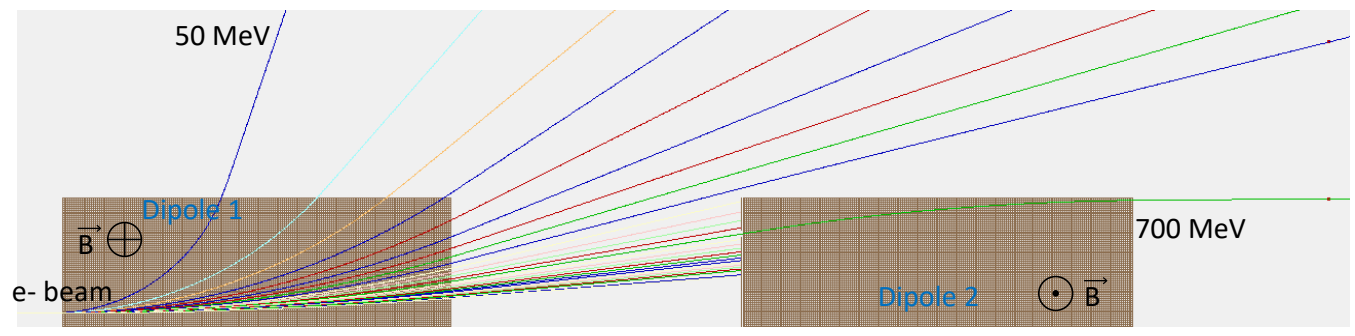

Figure 8. Sketch of the double dipole scheme used as an energy selector.

By inserting a collimator between both dipoles, the spectrometer can be used as an energy selector, as shown in Fig. 8. The energy and energy spread of the selected beam can be tuned by changing the position and the size of the collimator respectively. This gives an electron beam translated with respect to the laser axis with a potentially low-energy spread. This can be of interested for numerous applications such as the use of an imaging beam line to focus the electron beam on a target or to send a specific spectrally discriminated part of the beam to an undulator, for FEL experiments.

\subsection{Emittance measurement}

By measuring the electron beam energy and output angle of the bunch, the emittance can be measured employing the pepper pot technique, ${ }^{28,29}$ at a selected energy and even at high energies ${ }^{33,34}$ using the well-known relation for the RMS emittance $\epsilon_{r m s}: \epsilon_{r m s}=\sqrt{\left.\left\langle x^{2}\right\rangle<x^{\prime 2}\right\rangle-\left\langle x x^{\prime}\right\rangle^{2}}$, where $\left\langle x^{2}\right\rangle$ is the averaged square position, $\left\langle x^{\prime 2}\right\rangle$ is the averaged square divergence and $\left\langle x x^{\prime}\right\rangle$ the cross product. 


\subsection{Both dipoles with the same polarity}

Fig. 9 shows a sketch of the double dipole used with identical and same polarity magnetic fields a) and the error associated with different initial divergence as a function of the initial energy b).

a)

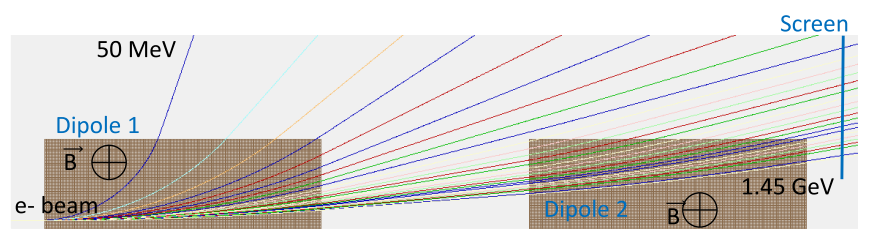

b)

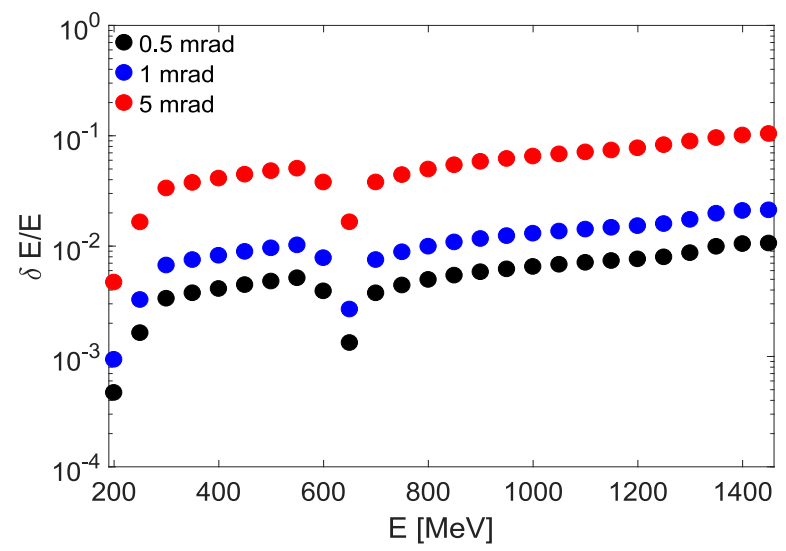

Figure 9. Sketch of the double dipole scheme with both dipoles with the same magnetic field and polarity a), error associated with different initial divergence angles as a function of the energy in logarithmic scale b).

This scheme allows to increase the energy resolution and lower the error on the energy determination. In this scheme again, discontinuities in the estimated error curve are seen due to the different trajectories associated with the different energies. This spectrometer configuration could be used along with a high F-number off-axis parabola and/or a long guiding target such as a capillary without ${ }^{35}$ or with a discharge ${ }^{36}$ enabling the production of higher energy electron beams.

\section{CONCLUSIONS}

In conclusion, we have showed that using a double dipole, double screen spectrometer, a very high precision energy measurement $(<1 \%$ at $1 \mathrm{GeV})$ can be achieved. This requires the differences between the two dipoles and the effect of the electron beam going through the first Lanex to be carefully taken into account. It also allows the electron trajectory to be traced back to determine the incoming angle of the electron in the first dipole which significantly reduces the error on the energy measurement and the broadening due to the beam divergence, thus enabling its effect to be simply de-convoluted.

\section{ACKNOWLEDGMENTS}

This work was partially supported by EuPRAXIA (Grant No. 653782), EC's LASERLAB-EUROPE (Grant No. 654148) and the UK Engineering and Physical Sciences Research Council (EPSRC) (Grant No. EP/N028694/1, $\mathrm{EP} / \mathrm{J} 018171 / 1, \mathrm{EP} / \mathrm{J} 500094 / 1, \mathrm{EP} / \mathrm{K} 011952 / 1$ and EP/P020607/1).

\section{REFERENCES}

[1] Tajima, T. and Dawson, J. M., "Laser Electron Accelerator," Physical Review Letters 43(4), 267-270 (1979).

[2] Geddes, C. G. R., Toth, C., van Tilborg, J., Esarey, E., Schroeder, C. B., Bruhwiler, D., Nieter, C., Cary, J., and Leemans, W. P., "High-quality electron beams from a laser wakefield accelerator using plasma-channel guiding," Nature 431, 538-541 (Sept. 2004).

[3] Faure, J., Glinec, Y., Pukhov, A., Kiselev, S., Gordienko, S., Lefebvre, E., Rousseau, J.-P., Burgy, F., and Malka, V., "A laserplasma accelerator producing monoenergetic electron beams," Nature 431, 541-544 (Sept. 2004). 
[4] Mangles, S. P. D., Murphy, C. D., Najmudin, Z., Thomas, A. G. R., Collier, J. L., Dangor, A. E., Divall, E. J., Foster, P. S., Gallacher, J. G., Hooker, C. J., Jaroszynski, D. A., Langley, A. J., Mori, W. B., Norreys, P. A., Tsung, F. S., Viskup, R., Walton, B. R., and Krushelnick, K., "Monoenergetic beams of relativistic electrons from intense laserplasma interactions," Nature 431, 535-538 (Sept. 2004).

[5] Leemans, W., Gonsalves, A., Mao, H.-S., Nakamura, K., Benedetti, C., Schroeder, C., Tth, C., Daniels, J., Mittelberger, D., Bulanov, S., Vay, J.-L., Geddes, C., and Esarey, E., "Multi-GeV Electron Beams from Capillary-Discharge-Guided Subpetawatt Laser Pulses in the Self-Trapping Regime," Physical Review Letters 113(24), 245002 (2014).

[6] Nakamura, K., Wan, W., Ybarrolaza, N., Syversrud, D., Wallig, J., and Leemans, W. P., "Broadband single-shot electron spectrometer for GeV-class laser-plasma-based accelerators," Review of Scientific Instruments 79, 053301 (May 2008).

[7] Sears, C. M. S., Cuevas, S. B., Schramm, U., Schmid, K., Buck, A., Habs, D., Krausz, F., and Veisz, L., "A high resolution, broad energy acceptance spectrometer for laser wakefield acceleration experiments," Review of Scientific Instruments 81, 073304 (July 2010).

[8] Valente, P., Anelli, F., Bacci, A., Batani, D., Bellaveglia, M., Benocci, R., Benedetti, C., Cacciotti, L., Cecchetti, C. A., Clozza, A., Cultrera, L., Di Pirro, G., Drenska, N., Faccini, R., Ferrario, M., Filippetto, D., Fioravanti, S., Gallo, A., Gamucci, A., Gatti, G., Ghigo, A., Giulietti, A., Giulietti, D., Gizzi, L. A., Koester, P., Labate, L., Levato, T., Lollo, V., Londrillo, P., Martellotti, S., Pace, E., Pathak, N., Rossi, A., Tani, F., Serafini, L., Turchetti, G., and Vaccarezza, C., "Development of a Multi-GeV spectrometer for laser-plasma experiment at FLAME," Nuclear Instruments and Methods in Physics Research Section A: Accelerators, Spectrometers, Detectors and Associated Equipment 653, 42-46 (Oct. 2011).

[9] Jaroszynski, D. A. and Vieux, G., "Coherent Radiation Sources Based on Laser Plasma Accelerators," AIP Conference Proceedings 647, 902-914 (Nov. 2002).

[10] Jaroszynski D.A, Bingham R, Brunetti E, Ersfeld B, Gallacher J, van der Geer B, Issac R, Jamison S.P, Jones D, de Loos M, Lyachev A, Pavlov V, Reitsma A, Saveliev Y, Vieux G, and Wiggins S.M, "Radiation sources based on laserplasma interactions," Philosophical Transactions of the Royal Society A: Mathematical, Physical and Engineering Sciences 364, 689-710 (Mar. 2006).

[11] Jaroszynski, D. A., Amnania, M. P., Aniculaesei, C., Battaglia, G., Brunetti, E., Chen, S., Cipiccia, S., Ersfeld, B., Gil, D. R., Grant, D. W., Grant, P., Hur, M. S., Gamiz, L. I. I., Kang, T., Kokurewicz, K., Kornaszewski, A., Li, W., Maitrallain, A., Manahan, G. G., Noble, A., Reid, L. R., Shahzad, M., Spesyvtsev, R., Subiel, A., Tooley, M. P., Vieux, G., Wiggins, S. M., Welsh, G. H., Yoffe, S. R., and Yang, X., "Compact radiation sources based on laser-driven plasma waves," in [XXII International Symposium on High Power Laser Systems and Applications], 11042, 110420Y, International Society for Optics and Photonics (Jan. 2019).

[12] Schlenvoigt, H.-P., Haupt, K., Debus, A., Budde, F., Jckel, O., Pfotenhauer, S., Schwoerer, H., Rohwer, E., Gallacher, J. G., Brunetti, E., Shanks, R. P., Wiggins, S. M., and Jaroszynski, D. A., "A compact synchrotron radiation source driven by a laser-plasma wakefield accelerator," Nature Physics 4, 130-133 (Feb. 2008).

[13] Wiggins, S. M., Anania, M. P., Welsh, G. H., Brunetti, E., Cipiccia, S., Grant, P. A., Reboredo-Gil, D., Manahan, G., Grant, D. W., and Jaroszynski, D. A., "Undulator radiation driven by laser-wakefield accelerator electron beams," in [Relativistic Plasma Waves and Particle Beams as Coherent and Incoherent Radiation Sources], 9509, 95090K, International Society for Optics and Photonics (May 2015).

[14] Loulergue, A., Labat, M., Evain, C., Benabderrahmane, C., Malka, V., and Couprie, M. E., "Beam manipulation for compact laser wakefield accelerator based free-electron lasers," New Journal of Physics 17(2), 023028 (2015).

[15] Anania, M. P., Brunetti, E., Wiggins, S. M., Grant, D. W., Welsh, G. H., Issac, R. C., Cipiccia, S., Shanks, R. P., Manahan, G. G., Aniculaesei, C., Geer, S. B. v. d., Loos, M. J. d., Poole, M. W., Shepherd, B. J. A., Clarke, J. A., Gillespie, W. A., MacLeod, A. M., and Jaroszynski, D. A., "An ultrashort pulse ultra-violet radiation undulator source driven by a laser plasma wakefield accelerator," Applied Physics Letters 104, 264102 (June 2014). 
[16] Couprie, M. E., Loulergue, A., Labat, M., Lehe, R., and Malka, V., "Towards a free electron laser based on laser plasma accelerators," Journal of Physics B: Atomic, Molecular and Optical Physics 47(23), 234001 (2014).

[17] Rao, B. S., Moorti, A., Rathore, R., Chakera, J. A., Naik, P. A., and Gupta, P. D., "High-quality stable electron beams from laser wakefield acceleration in high density plasma," Physical Review Special Topics Accelerators and Beams 17, 011301 (Jan. 2014).

[18] Maitrallain, A., Audet, T. L., Dobosz Dufrnoy, S., Chanc, A., Maynard, G., Lee, P., Mosnier, A., Schwindling, J., Delferrire, O., Delerue, N., Specka, A., Monot, P., and Cros, B., "Transport and analysis of electron beams from a laser wakefield accelerator in the $100 \mathrm{MeV}$ energy range with a dedicated magnetic line," $\mathrm{Nu}$ clear Instruments and Methods in Physics Research Section A: Accelerators, Spectrometers, Detectors and Associated Equipment 908, 159-166 (Nov. 2018).

[19] Weingartner, R., Fuchs, M., Popp, A., Raith, S., Becker, S., Chou, S. W., Heigoldt, M., Khrennikov, K., Wenz, J., Seggebrock, T., Zeitler, B., Major, Z., Osterhoff, J., Krausz, F., Karsch, S., and Grner, F., "Imaging laser-wakefield-accelerated electrons using miniature magnetic quadrupole lenses," Physical Review Special Topics - Accelerators and Beams 14 (May 2011).

[20] Thaury, C., Guillaume, E., Dpp, A., Lehe, R., Lifschitz, A., Ta Phuoc, K., Gautier, J., Goddet, J.-P., Tafzi, A., Flacco, A., Tissandier, F., Sebban, S., Rousse, A., and Malka, V., "Demonstration of relativistic electron beam focusing by a laser-plasma lens," Nature Communications 6, 6860 (Apr. 2015).

[21] van Tilborg, J., Barber, S., Tsai, H.-E., Swanson, K., Steinke, S., Geddes, C., Gonsalves, A., Schroeder, C., Esarey, E., Bulanov, S., Bobrova, N., Sasorov, P., and Leemans, W., "Nonuniform discharge currents in active plasma lenses," Physical Review Accelerators and Beams 20, 032803 (Mar. 2017).

[22] van der Geer, S. B., Luiten, O. J., de Loos, M. J., Pöplau, G., and van Rienen, U., "3d Space-charge model for GPT simulations of high-brightness electron bunches," Institute of Physics 175, 101-110 (2003).

[23] Chubar, O., Elleaume, P., and Chavanne, J., "A three-dimensional magnetostatics computer code for insertion devices," Journal of Synchrotron Radiation 5, 481-484 (May 1998).

[24] "Application programmes at the scottish centre for the application of plasma-based accelerators SCAPA," 11036, 11036-28 (2019). Proc. SPIE.

[25] Clayton, C. E., Ralph, J. E., Albert, F., Fonseca, R. A., Glenzer, S. H., Joshi, C., Lu, W., Marsh, K. A., Martins, S. F., Mori, W. B., Pak, A., Tsung, F. S., Pollock, B. B., Ross, J. S., Silva, L. O., and Froula, D. H., "Self-Guided Laser Wakefield Acceleration beyond $1 \mathrm{GeV}$ Using Ionization-Induced Injection," Physical Review Letters 105, 105003 (Sept. 2010).

[26] Soloviev, A. A., Starodubtsev, M. V., Burdonov, K. F., Kostyukov, I. Y., Nerush, E. N., Shaykin, A. A., and Khazanov, E. A., "Two-screen single-shot electron spectrometer for laser wakefield accelerated electron beams," Review of Scientific Instruments 82, 043304 (Apr. 2011).

[27] Pollock, B., Ross, J., Tynan, G., Clayton, C., Joshi, C., Marsh, K., Pak, A., Wang, T.-L., Divol, L., Froula, D., Glenzer, S., Leurent, V., Palastro, J., and Ralph, J., "Two-Screen Method for Determining Electron Beam Energy and Deflection from Laser Wakefield Acceleration," Conference PAC 2009, WE6RFP101 (Dec. 2010).

[28] Brunetti, E., Shanks, R. P., Manahan, G. G., Islam, M. R., Ersfeld, B., Anania, M. P., Cipiccia, S., Issac, R. C., Raj, G., Vieux, G., Welsh, G. H., Wiggins, S. M., and Jaroszynski, D. A., "Low Emittance, High Brilliance Relativistic Electron Beams from a Laser-Plasma Accelerator," Physical Review Letters 105, 215007 (Nov. 2010).

[29] Manahan, G. G., Brunetti, E., Aniculaesei, C., Anania, M. P., Cipiccia, S., Islam, M. R., Grant, D. W., Subiel, A., Shanks, R. P., Issac, R. C., Welsh, G. H., Wiggins, S. M., and Jaroszynski, D. A., "Characterization of laser-driven single and double electron bunches with a permanent magnet quadrupole triplet and pepper-pot mask," New Journal of Physics 16, 103006 (Oct. 2014). WOS:000344094100004.

[30] Moliere, G., "Theory of the scattering of fast charged particles. 2. Repeated and multiple scattering," $Z$. Naturforsch. A3, 78-97 (1948).

[31] Glinec, Y., Faure, J., Guemnie-Tafo, A., Malka, V., Monard, H., Larbre, J. P., Waele, V. D., Marignier, J. L., and Mostafavi, M., "Absolute calibration for a broad range single shot electron spectrometer," Review of Scientific Instruments 77, 103301 (Oct. 2006). 
[32] Battistoni, G., Boehlen, T., Cerutti, F., Chin, P. W., Esposito, L. S., Fass, A., Ferrari, A., Lechner, A., Empl, A., Mairani, A., Mereghetti, A., Ortega, P. G., Ranft, J., Roesler, S., Sala, P. R., Vlachoudis, V., and Smirnov, G., "Overview of the FLUKA code," Annals of Nuclear Energy 82, 10-18 (Aug. 2015).

[33] Delerue, N., Bartolini, R., Peach, K., Reichold, A., Senanayake, R., Bajlekov, S., Caballero-Bendixsen, L., Ibbotson, T., Thomas, C., Bourgeois, N., Walker, P. A., Buonomo, B., Mazzitelli, G., Doucas, G., Hooker, S., Lau, P., and Urner, D., "Single-Shot Emittance Measurement of a $508 \mathrm{MeV}$ Electron Beam Using the Pepper-Pot Method," Conference PAC 2009 , TH5RFP065 (Dec. 2010).

[34] Thomas, C., Delerue, N., and Bartolini, R., "Single shot $3 \mathrm{GeV}$ electron transverse emittance with a pepperpot," Nucl.Instrum.Meth. A729, 554-556 (July 2013).

[35] Desforges, F. G., Hansson, M., Ju, J., Senje, L., Audet, T. L., Dobosz-Dufrnoy, S., Persson, A., Lundh, O., Wahlstrm, C. G., and Cros, B., "Reproducibility of electron beams from laser wakefield acceleration in capillary tubes," Nuclear Instruments and Methods in Physics Research Section A: Accelerators, Spectrometers, Detectors and Associated Equipment 740, 54-59 (Mar. 2014).

[36] Gonsalves, A., Nakamura, K., Daniels, J., Benedetti, C., Pieronek, C., de Raadt, T., Steinke, S., Bin, J., Bulanov, S., van Tilborg, J., Geddes, C., Schroeder, C., Tth, C., Esarey, E., Swanson, K., Fan-Chiang, L., Bagdasarov, G., Bobrova, N., Gasilov, V., Korn, G., Sasorov, P., and Leemans, W., "Petawatt Laser Guiding and Electron Beam Acceleration to $8 \mathrm{GeV}$ in a Laser-Heated Capillary Discharge Waveguide," Physical Review Letters 122, 084801 (Feb. 2019). 